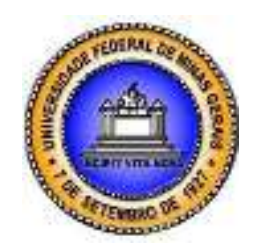

\title{
CONDIÇÕES E DESAFIOS AO DESENVOLVIMENTO DA GESTÃo DE DOCUMENTOS EM INSTITUIÇÕES DE SAÚDE NO BRASIL ${ }^{1}$
}

\section{CONDITIONS AND CHALLENGES IN THE DEVELOPMENT OF DOCUMENT MANAGEMENT IN HEALTH INSTITUTIONS IN BRAZIL}

\section{CONDICIONES Y DESAFÍOS DEL DESARROLLO DE LA GESTIÓN DE DOCUMENTOS EN INSTITUCIONES SANITARIAS DE BRASIL}

\author{
SILVIA APARECIDA PINHEIRO BRASIL DE SOUSA \\ Sinapses - Educação a Distância \\ silvia.brasil@sinapses-ead.com.br
}

\section{RESUMO}

A busca por soluções e padrões de eficiência no uso de documentos administrativos é pouco explorada na literatura, mas o conceito de memória organizacional e aprendizagem organizacional já podem ser encontrados na literatura dos estudos organizacionais e administrativos (SANTOS, 2012, p. 43 e SENGE 1990, apud CASTILHO; SILVA; TURRIONI, 2004, p.2). Pedrosa e Couto (2007, p.1) referem que as "organizações não suportarão mais custos relacionados com a má qualidade, tais como custos de retrabalho, custos com processos ineficientes, custos de eventuais processos nos organismos de defesa do consumidor ou outros fóruns legais," entre outros. O objetivo deste artigo é analisar, de forma sintética, a contribuição da avaliação externa para o fomento do tema gestão de documentos nos hospitais, bem como apresentar a homogeneidade e heterogeneidade das categorias de documentos requerida por esses. Trata-se de um estudo documental que visa identificar a ocorrência da citação de termos relacionados à estrutura documental de um sistema de gestão da qualidade, sob a óptica dos modelos de avaliação externa estudados. Destaca-se como ponto forte um conjunto de documentos comum aos três modelos de avaliação estudados.

PALAVRAS-CHAVE: Qualidade em saúde, Acreditação Hospitalar, Gestão do Conhecimento.

\begin{abstract}
The search for solutions and efficiency standards in the use of administrative documents is rarely considered in literature, but the concept of organizational memory and organizational learning can now be found in the literature on organizational and administrative studies (SANTOS, 2012, p.43 and SENGE 1990, with CASTILHO; SILVA; TURRIONI, 2004, p.2). Pedrosa and Couto $(2007$, p.1) narrate that the 'organizations won't handle more expenses related to the bad quality, such as costs with rework, inefficient processes, eventual lawsuits in the organisms of consumers' rights or other legal courthouses', among others. The aim of this article is to synthetically analyze the contribution of the external evaluation in the fomentation of the document management in hospitals theme, as well as to present homogeneity and

\footnotetext{
${ }^{1}$ Submetido em 05 de dezembro de 2013. Aceito em 20 de março de 2014. O artigo foi avaliado segundo o processo de duplo anonimato e avaliado pelos editores. Editores responsáveis: Márcio Augusto Gonçalves e Lucas Maia dos Santos. Reprodução parcial ou total e trabalhos derivativos permitidos com a citação apropriada da fonte.
} 

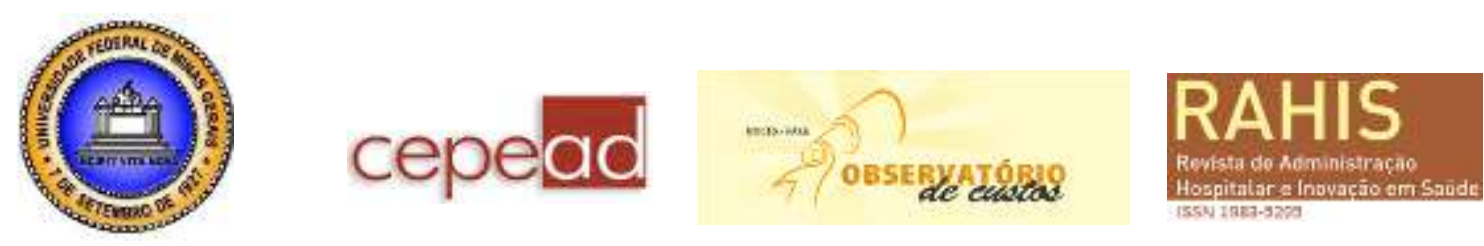

heterogeneity of the document categories required by them. It's a documental study that aims to identify the occurrence of the term references related to the documental structure of a system of quality management under the view of the studied external evaluation models. A set of documents common to the three studied evaluation models is highlighted as a strong point.

KEY WORDS: Health quality; Hospital accrediting; Knowledge management.

\section{RESUMEN}

La búsqueda de soluciones y estándares de eficiencia en el uso de documentos administrativos está poco explorada en la literatura, pero el concepto de memoria organizacional y aprendizaje organizacional ya pueden encontrarse en la literatura sobre los estudios organizacionales y administrativos (SANTOS, 2012, p. 43 y SENGE 1990, apud CASTILHO; SILVA; TURRIONI, 2004, p.2). Pedrosa y Couto (2007, p.1) refieren que las "organizaciones no soportarán más costos relacionados con la mala calidad, tales como costos de duplicación de trabajo, costos de procesos ineficientes, costos de eventuales procesos en los organismos de defensa del consumidor u otros foros legales," entre otros. El objetivo de este artículo es analizar, de forma sintética, la contribución de la evaluación externa al fomento del tema de la gestión de documentos en los hospitales, así como presentar la homogeneidad y heterogeneidad de las categorías de documentos requeridas por ellos. Se trata de un estudio documental que busca identificar la ocurrencia de la citación de términos relacionados con la estructura documental de un sistema de gestión de calidad, bajo la óptica de los modelos de evaluación externa estudiados. Se destaca como punto fuerte un conjunto de documentos común a los tres modelos de evaluación estudiados.

PALABRAS CLAVE: Calidad en la salud; Acreditación Hospitalera; Gestión del Conocimiento.

\section{Introdução}

Uma instituição de saúde que busca oferecer assistência com qualidade e segurança estabelece e documenta suas práticas. Ao estabelecer essas práticas, um conjunto de atividades será necessário para garantir o acesso à informação atualizada e aprovada pelas áreas técnicas competentes, envolvendo a padronização de categorias de documentos, o estabelecimento de prazos para revisão, vínculos e inter-relação formalizados com outros documentos institucionais, dentre outros. Esse conjunto de atividades é comumente denominado pelos profissionais da área da qualidade envolvidos nesse processo de gestão de documentos, embora esta prática não contemple a dimensão de recolhimento para guarda permanente, presente no conceito de gestão de documentos da arquivologia. Segundo o Dicionário Brasileiro de Terminologia Arquivística (2005) e o artigo 3 da Lei 8.159 (de 08 de janeiro de 1991), "considera-se gestão de documentos o conjunto de procedimentos e operações técnicas referentes à sua produção, à tramitação, ao uso, à avaliação e ao arquivamento em fase corrente e intermediária, visando a sua eliminação ou recolhimento para guarda permanente.”.

Em contrapartida, a literatura sobre a aplicação de princípios da administração científica para a solução de problemas documentais no contexto organizacional ainda é relativamente frágil, sobretudo, quanto à necessidade de se racionalizar e modernizar as administrações públicas de saúde.

Jardim refere não haver um consenso sobre qual a disciplina gestão de documentos estaria vinculada, se arquivística ou administração, e menciona que o historiador norte- 

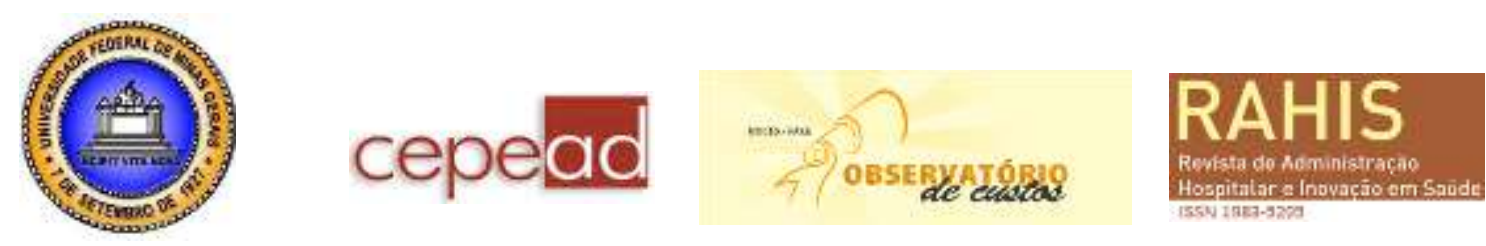

americano Lawrence Burnet relaciona a gestão de documentos a uma operação arquivística. $\mathrm{O}$ mesmo autor cita, ainda, o dicionário de terminologia arquivística, do Conselho Internacional de Arquivos, no qual "a gestão de documentos diz respeito a uma área da administração geral relacionada com a busca de economia e eficácia na produção, manutenção, uso e destinação final dos mesmos." (JARDIM, 1987, p.35).

Organizações prestadoras de serviços hospitalares

Segundo Pedrosa e Couto (2007, p.2), em 1957, a Organização Mundial da Saúde definiu hospital em seu Informe Técnico número 122, como:

Parte integrante de um sistema coordenado de saúde cuja função é dispensar à comunidade completa assistência à saúde, tanto curativa quanto preventiva, incluindo serviços extensivos à família, em seu domicílio e, ainda um centro de formação para os que trabalham no campo da saúde e para pesquisas biossociais.

A formação em Administração Hospitalar surgiu nos Estados Unidos, na Universidade de Chicago, em 1934. No Brasil, essa formação chegou em meados de 1946 (CAMARA e TEIXEIRA, 2011, p.1). Até este período, a administração se baseava em intuição e bom senso, segundo Pedrosa e Couto (2007, p.2 e 3), e esses atributos requerem, no mínimo experiência, o que leva tempo para aquisição, e, aqui vale ressaltar um dos 7 tipos de deficiência de aprendizagem organizacional, segundo Senge (2001, apud RICHE e MONTE ALTO), para o qual a aprendizagem com a experiência é uma ilusão, pois "nunca experimentamos diretamente as consequências das nossas ações mais importantes." (RICHE e MONTE ALTO, 2001, p.40).

O hospital é uma instituição complexa que reúne, ao mesmo tempo, equipes multiprofissionais oferecendo serviços simultâneos e diversificados nem sempre relacionados diretamente ao cuidado ao paciente, mas oferecendo suporte à melhoria do cuidado prestado. Essa complexidade ocasiona necessidade de regência por um arcabouço legal, dinâmico e variado (PEDROSA E COUTO, 2007, p.3) que requer o registro de todas as ações adequadamente para garantir a continuidade do cuidado, evidenciar o cumprimento de requisitos legais, além de proporcionar salvaguarda aos profissionais envolvidos, no caso de desfechos indesejáveis. Essa dinâmica permanente e continuamente registrada requer evidências científicas e também gera um volume de documentos, qualquer que seja o suporte adotado (digital ou convencional). Duas são as saídas deste processo: o prontuário do paciente, que é regulamentado por legislação específica, e as políticas, protocolos assistenciais e procedimentos que oferecem sustentação à linha de cuidado adotado pela instituição e pelas práticas administrativas.

A busca por soluções e padrões de eficiência no uso de documentos administrativos é pouco explorada na literatura, mas o conceito de memória organizacional e aprendizagem organizacional já podem ser encontrados na literatura dos estudos organizacionais e administrativos (SANTOS, 2012, p. 43 e SENGE 1990, apud CASTILHO, SILVA; TURRIONI, 2004, p.2). O conceito de memória organizacional, segundo Santos (2012, p. 43), "é utilizado em sentido metafórico, no intuito de explicar processos e práticas organizacionais para codificação, armazenamento e recuperação de informações e/ou conhecimentos.”. Stein (1995 apud SANTOS, 2012) argumenta que, ao não utilizar o conhecimento disponível (seja por razões políticas ou técnicas), a organização desperdiça recurso. Pedrosa e Couto (2007, p.1) referem que as "organizações não suportarão mais custos relacionados com à má qualidade, tais 

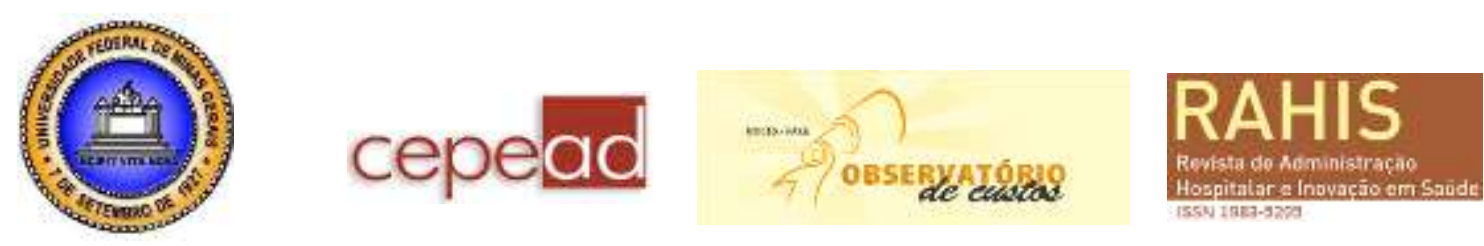

como custos de retrabalho, custos com processos ineficientes, custos de eventuais processos nos organismos de defesa do consumidor ou outros fóruns legais," entre outros. Segundo Almeida (2006, apud SANTOS, 2012), "o esquecimento ou falta de memória organizacional é dicotômico e por isso pode ser prejudicial à organização, principalmente quando ocorrem repetições de processos, projetos, ações ou até mesmo fracassos".

Pedrosa e Couto (2007, p.218) argumentam, ainda, que o hospital apresenta um elevadíssimo custo e:

[...] aproximadamente $70 \%$ do custo hospitalar é fixo, exigindo no planejamento ações para equacionar essa dificuldade. Sempre será necessário contemplar o tema estratégico da eficiência operacional, que inclui necessariamente o uso das ferramentas de mapeamento dos processos, a adequada interação desses processos, complementada pela descrição das tarefas e seguidos de capacitação de todo o nível operacional nos padrões de processo e tarefas.

O objetivo deste artigo é analisar, de forma sintética, a contribuição dos modelos de acreditação para o fomento do tema gestão de documentos nos hospitais, bem como, apresentar a homogeneidade e heterogeneidade das categorias de documentos requerida por cada modelo. Aspectos práticos para o estabelecimento de uma estrutura documental, do processo de edição à aprovação da documentação também serão abordados.

Gestão de documentos, um diferencial de organização

A gestão de documentos possui raízes no final do século XIX quando EUA e Canadá enfrentavam problemas de uso e guarda de documentos na administração pública. Comissões governamentais foram criadas com a finalidade de propor soluções e padrões de eficiência no uso de documentos, um dos aspectos mais importantes da Arquivologia, que se desenvolveu como uma disciplina a partir da segunda metade do século XIX. "Vale ressaltar que, durante esse período, as instituições arquivísticas públicas caracterizavam-se pela sua função de órgãos estritamente de apoio à pesquisa comprometidos com a conservação e acesso aos documentos considerados de valor histórico.”. (JARDIM, 1987, p.36).

Os chamados "documentos administrativos" não eram contemplados por esta concepção, por isso iniciativas da administração pública.

Paralelamente, iniciava-se a era da chamada "administração científica", em que Frederick Taylor e Frank Gilbreth procuravam mostrar aos administradores como racionalizar o processo administrativo, desenvolvendo suas atividades de forma menos dispendiosa, melhor e mais rápida. [...] A aplicação dos princípios da administração científica para a solução dos problemas documentais gerou os princípios gestão de documentos, os quais resultaram, sobretudo, da necessidade de se racionalizar e modernizar as administrações. (JARDIM, 1987, p.36).

A gestão de documentos ainda raramente é vista nas instituições de saúde brasileiras, mas, por ser uma solução aplicável à gestão da qualidade, começa a ganhar notoriedade.

Segundo Pedrosa e Couto (2007, p.306), as metodologias de avaliação externa de qualidade aplicáveis a hospitais disponíveis no Brasil são a certificação e a acreditação. Ambas são de caráter voluntário.

O modelo de certificação, norma sistêmica de gestão e garantia da qualidade é representado pela International Organization for Standardization (ISO) e o modelo de acreditação, cuja "norma sistêmica de gestão e garantia da qualidade mais itens de 

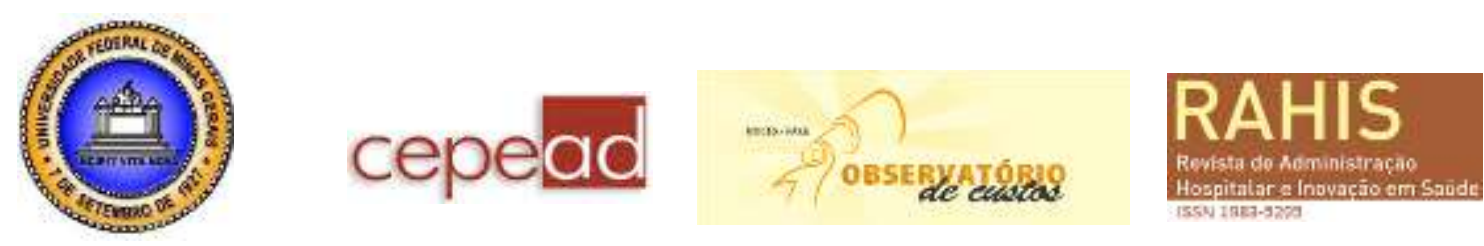

funcionamento específicos para hospital" (PEDROSA E COUTO, 2007, p.306) é representado pela Organização Nacional de Acreditação (ONA), o Programa Compromisso com a Qualidade Hospitalar (CQH) e o Consórcio Brasileiro de Acreditação (CBA).

$\mathrm{O}$ modelo de acreditação nasceu e evoluiu no setor da saúde. O CQH se inspirou nos trabalhos da Comissão Conjunta de Acreditação de Organizações de Saúde (CCAOS), dos EUA. A ONA surgiu de iniciativas regionais relacionadas diretamente com a acreditação hospitalar. Os modelos brasileiros são novos quando comparados aos modelos internacionais, ma, devido às referências utilizadas, já apresentam em seu escopo a ênfase para o tema "padrões de trabalho" e "documentação".

Para o modelo de avaliação proposto pelo programa do $\mathrm{CQH}$, os padrões de trabalho podem ser estabelecidos "[...] tomando como critérios as necessidades das partes interessadas, as estratégias, requisitos legais, nível de desempenho de concorrentes, informações comparativas pertinentes, normas nacionais e internacionais, entre outros." (Roteiro de Visitas 10-01, p.47).

Para o modelo de avaliação proposto pela Organização Nacional de Acreditação (ONA), a gestão de documentos é um requisito do padrão de nível 1, na versão 2010 do Manual Brasileiro de Acreditação, instrumento de avaliação, publicado pela instituição citada. Na versão anterior (publicada em 2006), a gestão de documentos era contemplada nos itens de orientações do padrão de nível 2, com a seguinte redação: "documentação (procedimentos e registros) atualizada, disponível e aplicada". Esta mudança, remanejamento do item de orientação do padrão de nível 2 para nível 1, considerado requisito mínimo para se obter a acreditação, aliada a uma nova redação do item de orientação "Gestão de documentos", pode indicar maior rigor no padrão para avaliação com relação aos documentos. Esta mudança também pode evidenciar o empenho da ONA em promover a gestão profissional e padrões de eficiência no uso de documentos nas instituições de saúde brasileiras.

Vale ressaltar que, em 2006, segundo informações publicadas em seu web site, a ONA atingiu a marca de 125 diagnósticos organizacionais realizados, 73 avaliações para a certificação e 66 certificados concedidos. E possível verificar a publicação de dados nesse formato até 2009 .

O modelo de acreditação da Joint Commission International (JCI) apresenta padrões com foco no paciente e com foco em administração de instituições de saúde. Cada padrão apresenta propósitos e elementos de mensuração, e ambos apresentam, em sua redação, a necessidade de políticas, procedimentos, programas e planos. Um programa de melhoria da qualidade e segurança do paciente também é requerido. Os documentos citados devem apresentar uma inter-relação, sendo que os procedimentos e protocolos darão sustentabilidade às políticas, e os planos evidenciarem o cumprimento dos programas.

As equipes envolvidas no desenvolvimento e gestão de documentos

A maior aliada da gestão de documentos são as equipes envolvidas na edição e revisão da documentação, pois não basta iniciar, é imprescindível manter a documentação atualizada. Segundo Roncaglio, Svarza e Bojanov (2004, p. 5), "a definição dos recursos humanos empregados nos arquivos de empresas públicas ou privadas está relacionada à complexidade da atividade da instituição, ao volume do acervo e à periodicidade de revisão dos documentos". Esse requisito pode ser aplicado ao desenvolvimento de um sistema de gestão da qualidade e às atividades correlatas à gestão de documentos. 

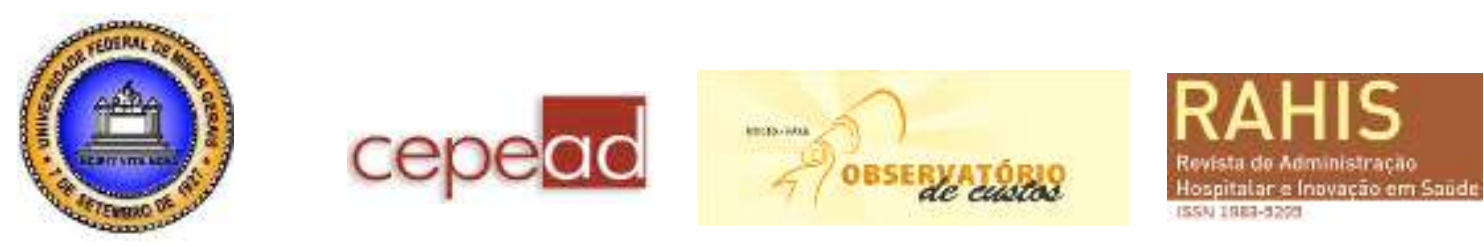

Para a seleção dos envolvidos na edição e revisão da documentação, é recomendável a identificação de habilidades com recursos tecnológicos e domínio cognitivo para correlacionar atividades, processos e resultados. A capacitação das equipes para o desenvolvimento da documentação deve se basear na pedagogia da problematização, pois, segundo Pedrosa e Couto (2007, p.168), essa é a metodologia que irá propiciar a transformação efetiva do trabalho e das instituições por estimular a reflexão crítica da prática e da identificação de problemas, e proposição de hipóteses para melhorias e correções nos processos.

Segundo Roncaglio, Svarza e Bojanov (2004, p. 5):

[...] a operacionalização da gestão de documentos, isto é, a organização interna do trabalho, conta muito nos resultados obtidos. Por trás das metodologias escolhidas e das normas implantadas deve existir um conjunto de pessoas com competências e habilidades variadas, pensando e atuando juntas para que o fluxo e a tramitação dos documentos, os assuntos selecionados, os prazos definidos, os cuidados de conservação estabelecidos e o arquivamento final espelhem a realidade institucional ou contribuam para a sua interpretação.

Mas capturar o conhecimento informal requer muita habilidade dos líderes, pois, segundo Santos (2012, p. 44), a "existência de barreiras tecnológicas, culturais e políticas para o desenvolvimento de mecanismos capazes de capturar o conhecimento informal, tornando-o explícito, são relevantes para análise da memória organizacional" e podemos acrescentar que é um dos maiores desafios desse processo, assim como afirma Coklin (2001 apud SANTOS, 2012):

Entre as barreiras apontadas anteriormente, destacam-se: a existência do conhecimento informal e a extrema dificuldade na sua captura e transformação; a prática na preservação de documentos, cujo foco está no armazenamento, sem preservação do contexto no qual o conhecimento foi produzido ou utilizado; a perda de relevância de determinado conhecimento após seu uso; [...]

A área da qualidade tem um papel fundamental ao atuar como um guardião do padrão da documentação, promovendo a capacitação dos envolvidos, estimulando a revisão e alertando para as conexões necessárias culminando com a manutenção do sistema como um organismo vivo dentro da instituição, além de explicitar o contexto registrando os períodos de mudanças e respectivos gatilhos.

A aplicação de tecnologia para a gestão de documentos não se configura apenas em aquisição e uso de softwares específicos disponíveis no mercado, ou desenvolvidos por profissionais da instituição, mas no desenvolvimento de um sistema de gestão da qualidade com definição de categorias e tipos de documentos, respectivos objetivos, modelos padronizados e orientações para elaboração, além de delinear a hierarquia e inter-relação entre os documentos.

\section{Metodologia}

Trata-se de um estudo documental que visa identificar as ocorrências de termos relacionados à estrutura documental de um sistema de gestão da qualidade, sob a óptica dos modelos de avaliação externa estudados, sua homogeneidade e heterogeneidade.

Os documentos selecionados estão especificados no Quadro 1.

\section{Descrição do Corpus}


Serão analisados os padrões para avaliação da qualidade de três modelos de avaliação externa de hospitais, todos já aplicados aqui no Brasil. Os padrões de avaliação possuem uma heterogeneidade de propostas em relação à estrutura e organização do instrumento, em relação à metodologia utilizada na avaliação e dinâmica da visita de avaliação. Os instrumentos selecionados correspondem às edições atualmente em uso no Brasil, com menos de cinco anos de publicação, ou seja, ainda recentes.

O Quadro 1, abaixo, esquematiza de forma sintética os padrões escolhidos, os objetivos do programa, editora que os edita, a data da publicação e a versão.

Quadro 1 - Corpus dos padrões de avaliação

\begin{tabular}{|c|c|c|c|c|}
\hline Título & $\begin{array}{c}\text { Objetivo } \\
\text { (do programa) }\end{array}$ & Editora & Data & Versão/Edição \\
\hline $\begin{array}{l}\text { Roteiro de } \\
\text { Visitas }\end{array}$ & $\begin{array}{c}\text { Contribuir para a } \\
\text { melhoria } \\
\text { contínua da } \\
\text { qualidade } \\
\text { hospitalar }\end{array}$ & $\begin{array}{l}\text { (documento } \\
\text { em pdf) }\end{array}$ & $\begin{array}{c}\text { Março de } \\
2011\end{array}$ & $10-01$ \\
\hline $\begin{array}{c}\text { Padrões de } \\
\text { Acreditação da } \\
\text { Joint } \\
\text { Commission } \\
\text { International } \\
\text { para Hospitais }\end{array}$ & $\begin{array}{c}\text { Estimular a } \\
\text { demonstração de } \\
\text { uma melhoria } \\
\text { contínua e } \\
\text { sustentada nas } \\
\text { instituições de } \\
\text { saúde }\end{array}$ & $\begin{array}{c}\text { Consórcio } \\
\text { Brasileiro de } \\
\text { Acreditação de } \\
\text { Sistemas e } \\
\text { Serviços de } \\
\text { Saúde, Rio de } \\
\text { Janeiro }\end{array}$ & $\begin{array}{c}\text { Janeiro de } \\
2011\end{array}$ & $4^{\mathrm{a}}$ edição \\
\hline $\begin{array}{c}\text { Manual } \\
\text { Brasileiro de } \\
\text { Acreditação-- } \\
\text { Organizações } \\
\text { Prestadoras de } \\
\text { Serviços de } \\
\text { Saúde }\end{array}$ & $\begin{array}{c}\text { Implantação e } \\
\text { implementação } \\
\text { em nível nacional } \\
\text { de um processo } \\
\text { permanente de } \\
\text { melhoria da } \\
\text { qualidade da } \\
\text { assistência à } \\
\text { saúde. }\end{array}$ & $\begin{array}{l}\text { Organização } \\
\text { Nacional de } \\
\text { Acreditação, } \\
\text { Brasília }\end{array}$ & $\begin{array}{c}\text { Março de } \\
2010\end{array}$ & 2010 \\
\hline
\end{tabular}

Fonte: Elaboração própria.

É importante ressaltar que o Manual Brasileiro de Acreditação e o Programa Compromisso com a Qualidade Hospitalar são iniciativas brasileiras baseadas no instrumento da OPAS - Organização Panamericana de Saúde e nos trabalhos da Comissão Conjunta de Acreditação de Organizações de Saúde (CCAOS) dos EUA, respectivamente.

Após a análise geral dos padrões de avaliação, foi realizada a análise de conteúdo, que pressupõe um processo de redução de dados. Para chegar a elementos que permitissem estabelecer relações e obter conclusões, foi realizada uma separação em unidades relevantes e significativas para o tema selecionado, gestão de documentos. Essa divisão da informação em unidades foi realizada segundo as categorias de documento.

Para este trabalho, utilizamos a definição de documento apresentada por Roncaglio, Svarza e Bojanov (2004, p. 2), para os quais documento é a informação produzida e/ou acumulada 
"organicamente no decorrer das atividades de uma pessoa, família, instituição pública ou privada. Ou seja, os documentos são criados uns após os outros, em decorrência das necessidades sociais e legais da sociedade e do próprio desenvolvimento da vida pessoal ou institucional.”.

Procedeu-se, então, a leitura dos padrões selecionados para verificar a ocorrência de termos relacionados a categorias de documentos para o estabelecimento de um sistema de classificação, que, segundo Silva e Rocha (2010, p.746), se constrói "a partir da identificação e contraste de diversidades e uniformidades". O registro das citações foi organizado nos Quadros de 02 a 04 obedecendo-se à estrutura de cada padrão analisado.

Quadro 2 - Documentação requerida - metodologia CQH

Roteiro de Visitas CQH - versão 10-01

\begin{tabular}{|c|c|c|c|c|c|c|c|c|c|c|}
\hline \multirow{2}{*}{\multicolumn{2}{|c|}{ Estrutura do Roteiro }} & \multicolumn{9}{|c|}{ Ocorrência de citação dos documentos } \\
\hline & & \multirow{2}{*}{ 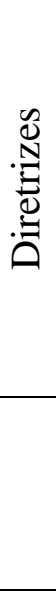 } & \multirow{2}{*}{ 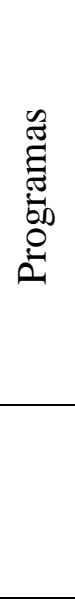 } & \multirow{2}{*}{$\frac{\mathscr{0}}{\stackrel{\Xi}{a}}$} & \multirow{2}{*}{$\begin{array}{l}0 \\
\text { ঠे } \\
\delta \\
\delta \\
0 \\
0\end{array}$} & \multirow{2}{*}{ 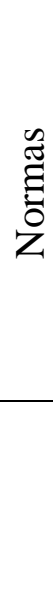 } & \multirow{2}{*}{ 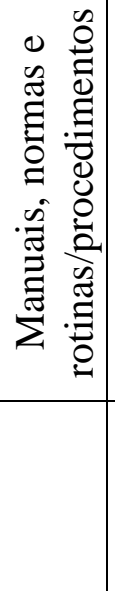 } & \multirow{2}{*}{ 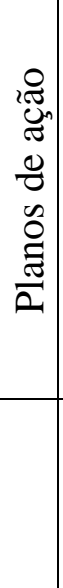 } & \multirow{2}{*}{ 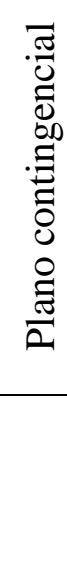 } & \multirow{2}{*}{$\begin{array}{l}0 \\
\frac{0}{0} \\
0 \\
0 \\
0 \\
0\end{array}$} \\
\hline \multirow[t]{11}{*}{$\begin{array}{l}\text { Instruções } \\
\text { gerais para } \\
\text { a visita }\end{array}$} & $\begin{array}{l}\text { Documentação obrigatória à } \\
\text { realização da visita I (licença } \\
\text { de funcionamento, } \\
\text { organograma e outros) }\end{array}$ & & & & & & & & & \\
\hline & - Critério VIII Resultados & & $\Delta$ & & $\Delta$ & & & & & \\
\hline & $\begin{array}{l}\text { Documentação para análise } \\
\text { no início da visita }\end{array}$ & & & & & & & & & \\
\hline & - Perfil & & & & & & & & & \\
\hline & - Critério I Liderança & & & & & & $\Delta$ & & & \\
\hline & $\begin{array}{l}\text { - Critério II Estratégias e } \\
\text { Planos }\end{array}$ & & & & & & & $\mathbf{\Delta}$ & & \\
\hline & - Critério III Clientes & & & & & & & & & \\
\hline & - Critério IV Sociedade & & & $\boldsymbol{\Delta}$ & & & & & & \\
\hline & - Critério VI Pessoas & & & & & & & & & \\
\hline & - Critério VII Processos & & $\Delta$ & & & $\boldsymbol{\Delta}$ & $\Delta$ & & $\boldsymbol{\Delta}$ & $\Delta$ \\
\hline & Selo de Conformidade & & & & $\mathbf{\Delta}$ & & & & & \\
\hline \multirow[t]{2}{*}{ Critérios } & Perfil & & & & & $\Delta$ & & & & \\
\hline & I. Liderança & $\mathbf{\Delta}$ & $\Delta$ & & & $\boldsymbol{\Delta}$ & $\Delta$ & $\boldsymbol{\Delta}$ & & \\
\hline
\end{tabular}




\begin{tabular}{|c|c|c|c|c|c|c|c|c|c|}
\hline \multirow[b]{3}{*}{ Estrutura do Roteiro } & CQI & $-\mathrm{v}$ & sãc & -0 & & & & & \\
\hline & \multicolumn{9}{|c|}{ Ocorrência de citação dos documentos } \\
\hline & 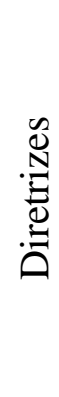 & 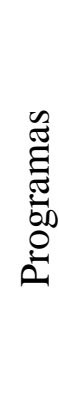 & 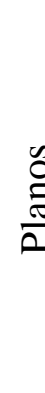 & $\begin{array}{l}0 \\
\mathscr{d} \\
0 \\
0 \\
0\end{array}$ & है & 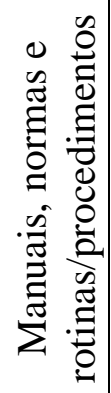 & 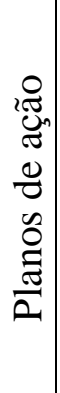 & 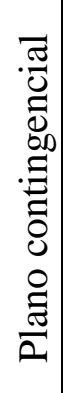 & $\begin{array}{l}\text { o } \\
0 \\
0 \\
0 \\
0 \\
0 \\
0\end{array}$ \\
\hline II. Estratégias e Planos & & & $\Delta$ & $\Delta$ & & & $\Delta$ & & \\
\hline III. Clientes & & & & $\Delta$ & & & & & \\
\hline IV. Sociedade & & $\mathbf{\Delta}$ & & $\Delta$ & & & & & \\
\hline $\begin{array}{l}\text { V. Informações e } \\
\text { Conhecimento }\end{array}$ & & $\mathbf{\Delta}$ & & $\Delta$ & & $\Delta$ & & & $\boldsymbol{\Delta}$ \\
\hline VI. Pessoas & $\Delta$ & $\Delta$ & $\Delta$ & $\Delta$ & & & & & \\
\hline VII. Processos & & & $\Delta$ & $\Delta$ & & $\boldsymbol{\Delta}$ & $\boldsymbol{\Delta}$ & & $\Delta$ \\
\hline
\end{tabular}

Fonte: Elaboração própria.

Quadro 3 - Documentação requerida - metodologia ONA

Manual das Organizações Prestadoras de Serviços de Saúde - ONA

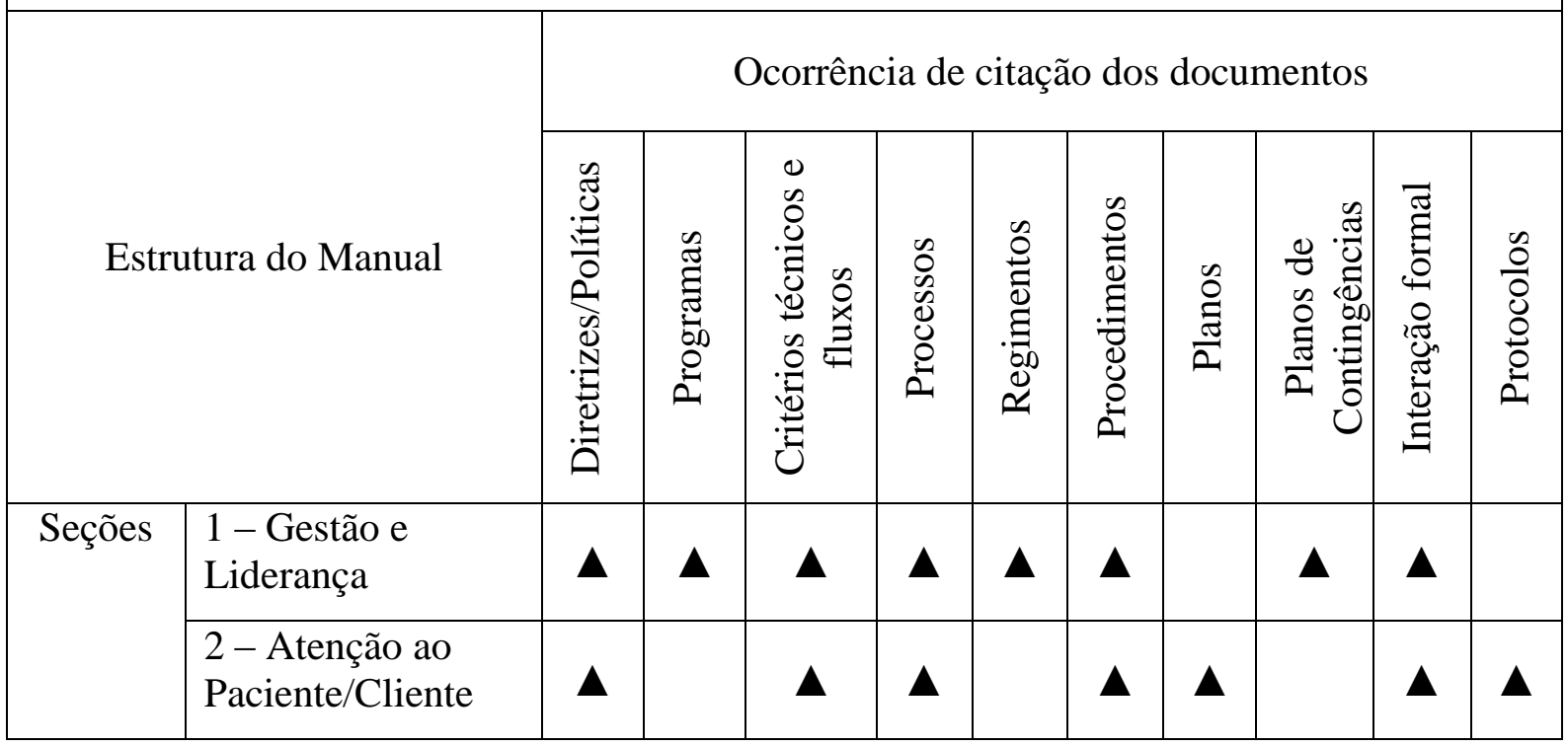




\begin{tabular}{|c|c|c|c|c|c|c|c|c|c|}
\hline 3 - Diagnóstico & $\Delta$ & & $\Delta$ & $\boldsymbol{\Delta}$ & $\Delta$ & $\Delta$ & $\boldsymbol{\Delta}$ & $\Delta$ & $\boldsymbol{\Delta}$ \\
\hline 4-Apoio Técnico & $\mathbf{\Delta}$ & $\Delta$ & $\Delta$ & $\boldsymbol{\Delta}$ & $\mathbf{\Delta}$ & & $\boldsymbol{\Delta}$ & $\Delta$ & \\
\hline $\begin{array}{l}5 \text { - Abastecimento } \\
\text { e Apoio Logístico }\end{array}$ & $\Delta$ & $\Delta$ & $\Delta$ & $\boldsymbol{\Delta}$ & $\boldsymbol{\Delta}$ & $\boldsymbol{\Delta}$ & $\boldsymbol{\Delta}$ & $\boldsymbol{\Delta}$ & $\boldsymbol{\Delta}$ \\
\hline 6 - Infraestrutura & & & & & & & $\boldsymbol{\Delta}$ & $\boldsymbol{\Delta}$ & \\
\hline
\end{tabular}

Fonte: Elaboração própria.

Quadro 4 - Documentação requerida - metodologia JCI

Padrões de Acreditação da Joint Comission International para Hospitais

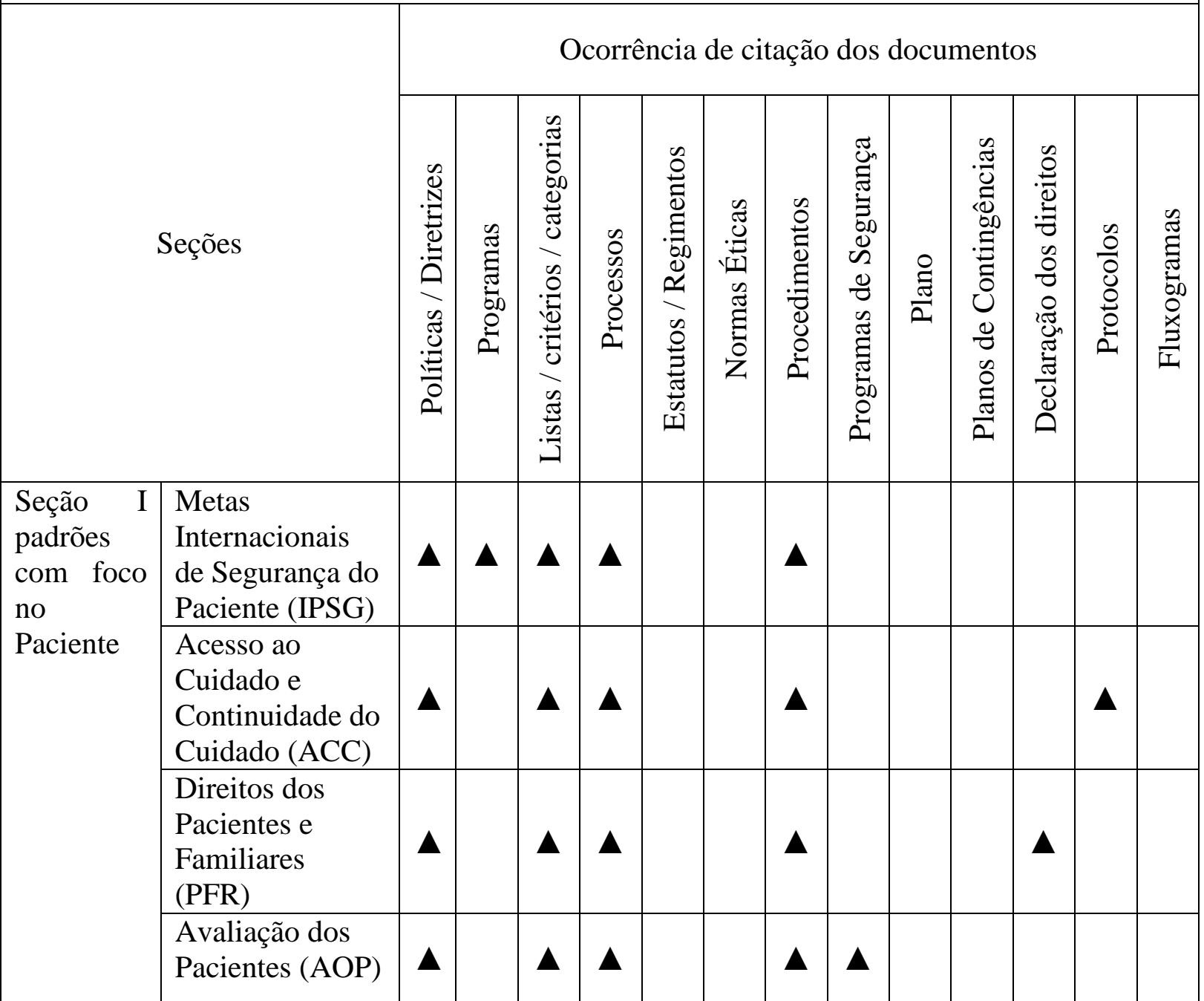




\begin{tabular}{|c|c|c|c|c|c|c|c|c|c|c|c|c|c|c|}
\hline \multicolumn{15}{|c|}{ Padrões de Acreditação da Joint Comission International para Hospitais } \\
\hline \multirow{2}{*}{\multicolumn{2}{|c|}{ Seções }} & \multicolumn{13}{|c|}{ Ocorrência de citação dos documentos } \\
\hline & & 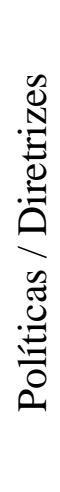 & 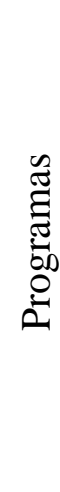 & 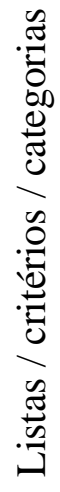 & $\begin{array}{l}0 \\
0 \\
0 \\
0 \\
0 \\
0 \\
0\end{array}$ & 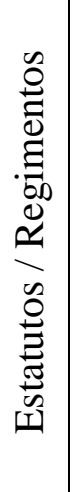 & 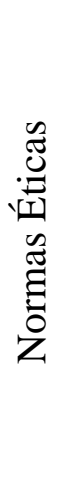 & 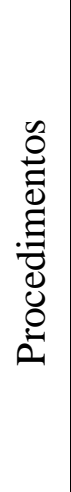 & 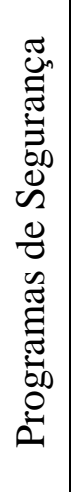 & $\frac{\stackrel{\varrho}{\Xi}}{\bar{\alpha}}$ & 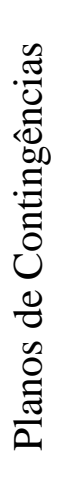 & 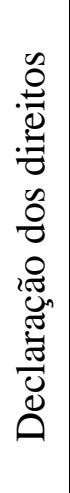 & $\begin{array}{l}0 \\
0 \\
0 \\
0 \\
0 \\
0 \\
0 \\
0\end{array}$ & 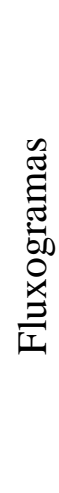 \\
\hline & $\begin{array}{l}\text { Cuidado aos } \\
\text { Pacientes (COP) }\end{array}$ & $\Delta$ & & & $\Delta$ & & & $\Delta$ & & & & & & $\Delta$ \\
\hline & $\begin{array}{l}\text { Anestesia e } \\
\text { Cirurgia (ASC) }\end{array}$ & $\Delta$ & & $\Delta$ & & & & $\boldsymbol{\Delta}$ & & & & & & \\
\hline & $\begin{array}{l}\text { Gerenciamento } \\
\text { e Uso de } \\
\text { Medicamentos } \\
\text { (MMU) }\end{array}$ & $\Delta$ & & $\mathbf{\Delta}$ & $\Delta$ & & & $\Delta$ & & & & & & \\
\hline & $\begin{array}{l}\text { Educação de } \\
\text { Pacientes e } \\
\text { Familiares } \\
(\text { PFE) }\end{array}$ & & & & $\Delta$ & & & & & & & & & \\
\hline \multirow{5}{*}{$\begin{array}{l}\text { Seção II: } \\
\text { Padrões } \\
\text { de } \\
\text { Adminis- } \\
\text { tração de } \\
\text { Institui- } \\
\text { ções de } \\
\text { Saúde }\end{array}$} & $\begin{array}{l}\text { Melhoria da } \\
\text { Qualidade e } \\
\text { Segurança do } \\
\text { Paciente (QPS) }\end{array}$ & $\Delta$ & $\boldsymbol{\Delta}$ & & $\Delta$ & & & & $\Delta$ & $\Delta$ & & & $\Delta$ & $\boldsymbol{\Delta}$ \\
\hline & $\begin{array}{l}\text { Prevenção e } \\
\text { Controle de } \\
\text { Infecções (PCI) }\end{array}$ & $\mathbf{\Delta}$ & $\mathbf{\Delta}$ & $\mathbf{\Delta}$ & $\mathbf{\Delta}$ & & & $\mathbf{\Delta}$ & & $\mathbf{\Delta}$ & & & $\Delta$ & \\
\hline & $\begin{array}{l}\text { Governo, } \\
\text { Liderança e } \\
\text { Direção (GLD) }\end{array}$ & $\mathbf{\Delta}$ & $\boldsymbol{\Delta}$ & $\mathbf{\Delta}$ & $\mathbf{\Delta}$ & $\Delta$ & $\boldsymbol{\Delta}$ & $\Delta$ & & $\Delta$ & & & & \\
\hline & $\begin{array}{l}\text { Gerenciamento } \\
\text { e Segurança das } \\
\text { Instalações } \\
(\mathrm{FMS})\end{array}$ & $\mathbf{\Delta}$ & $\boldsymbol{\Delta}$ & $\boldsymbol{\Delta}$ & $\Delta$ & & & $\mathbf{\Delta}$ & & $\Delta$ & & & & \\
\hline & $\begin{array}{l}\text { Educação e } \\
\text { Qualificação de }\end{array}$ & $\Delta$ & $\boldsymbol{\Delta}$ & & $\Delta$ & & & $\boldsymbol{\Delta}$ & & $\Delta$ & & & & \\
\hline
\end{tabular}



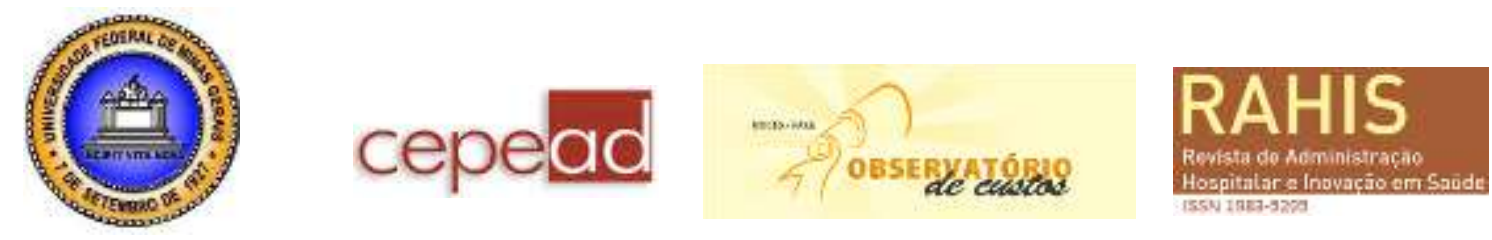

Padrões de Acreditação da Joint Comission International para Hospitais

\begin{tabular}{|c|c|c|c|c|c|c|c|}
\hline \multirow[b]{2}{*}{ Seções } & \multicolumn{7}{|c|}{ Ocorrência de citação dos documentos } \\
\hline & 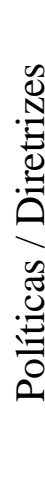 & 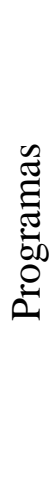 & 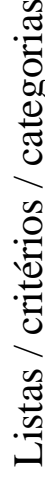 & $\begin{array}{l}0 \\
0 \\
y \\
y \\
y \\
0 \\
0 \\
0\end{array}$ & & & 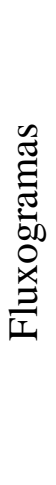 \\
\hline $\begin{array}{l}\text { Profissionais } \\
\text { (SQE) }\end{array}$ & & & & & & & \\
\hline $\begin{array}{l}\text { Gerenciamento } \\
\text { da Comunicação } \\
\text { e Informação } \\
\text { (MCI) }\end{array}$ & $\boldsymbol{\Delta}$ & & $\boldsymbol{\Delta}$ & $\Delta$ & & & \\
\hline
\end{tabular}

Fonte: Elaboração própria.

Cabe ressaltar alguns aspectos importantes: missão, visão e valores, estão incluídos na categoria "diretrizes". O padrão de avaliação da JCI enfatiza que os documentos devem estar de acordo com as leis, os regulamentos e as práticas aceitas atualmente, e que políticas e procedimentos devem estar implementados. Neste estudo, não estão inclusos os formulários.

A Tabela 1 apresenta os dados consolidados em três segmentos: categorias de documentos que são requeridas pelos três modelos de avaliação, categorias de documentos que são requeridas por apenas dois modelos e categorias de documentos específicas de cada modelo de avaliação. No primeiro segmento, identificamos a homogeneidade e, no terceiro segmento, as heterogeneidades.

\section{Os desafios ao desenvolvimento da gestão de documentos}

Relatos de experiências (informação verbal) ${ }^{2}$ com a gestão de documentos em instituições em busca de certificação (NBR ISO 9001/2000) ou acreditação nacional (ONA) sem o uso de tecnologia apropriada reforçam a limitação da abrangência a um pequeno número de participantes ou de categorias de documentos tornando-se necessário o enfrentamento de desafios correspondentes às diversas fases do processo de edição de documentos descritos no Quadro 5, e um profundo conhecimento dos elementos característicos da administração de instituições de saúde, quais sejam: "sua complexidade, diversidade, evolução e, sobretudo, suas demandas". (JARDIM, 1987, p.41).

\footnotetext{
${ }^{2}$ Relatos informais colhidos pela a autora durante a participação em curso sobre Abordagem por Processos, em São Paulo, em outubro de 2005.
} 


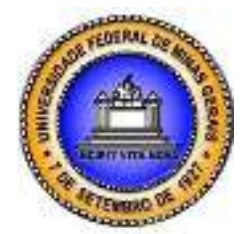

Quadro 5 - Fases do processo de edição de documentos e desafios identificados

\begin{tabular}{|l|l|}
\hline \multicolumn{1}{|c|}{ Fases } & \multicolumn{1}{|c|}{ Descrição dos desafios } \\
\hline Edição & $\begin{array}{l}\text { Manutenção da apresentação gráfica (fonte, margem, } \\
\text { espaçamento e paginação) e versão inicial do documento } \\
\text { estabelecido institucionalmente. Se houver diferenças neste } \\
\text { requisito entre as categorias de documento, maior será o } \\
\text { desafio. }\end{array}$ \\
\hline Ciclo de aprovação & $\begin{array}{l}\text { Dificuldade de verificação dos envolvidos durante o processo } \\
\text { de revisão aprovação. } \\
\text { Dificuldade de localização do documento, bem como, no } \\
\text { monitoramento do tempo empregado nesta fase pelos } \\
\text { envolvidos. } \\
\text { Documentos aprovados sem a revisão recomendada das áreas } \\
\text { técnicas, como, por exemplo: do Serviço de Controle de } \\
\text { Infecção Hospitalar (SCIH). }\end{array}$ \\
\hline Registros & $\begin{array}{l}\text { Ausência de meios para registro da análise da área da } \\
\text { qualidade e demais áreas técnicas envolvidas. } \\
\text { Impossibilidade de registro de sugestões de melhorias para as } \\
\text { próximas revisões. }\end{array}$ \\
\hline Vigência & $\begin{array}{l}\text { Estabelecimento de processo para identificação de cópias } \\
\text { controladas e não controladas. } \\
\text { Processo de controle e distribuição de cópias físicas aos } \\
\text { setores, trabalhoso e dispendioso. } \\
\text { Documento original na área emitente sem registro no acervo } \\
\text { institucional. } \\
\text { Documentos sem aprovação dos profissionais indicados em } \\
\text { meio aos documentos vigentes, induzindo operadores à } \\
\text { utilização sem respaldo técnico. }\end{array}$ \\
\hline
\end{tabular}

Elaborado pela autora.

As consultas e pesquisas aos documentos vigentes se constituem em um desafio para além do processo de edição quando não for empregada tecnologia apropriada. A consulta a um documento somente será possível se o interessado estiver na área emitente ou área de aplicação do documento, nas quais haverá uma cópia controlada, e, na área centralizadora do acervo original, normalmente a área da qualidade, em que deverão constar todos os documentos originais. Dependendo da forma instituída para controle do acervo, será possível consultar apenas por palavra-chave no título do documento. Esse obstáculo poderia ser facilmente superado se os idealizadores da estrutura documental propusessem um campo para palavrachave, devendo essas ser contempladas no controle do acervo e devidamente identificadas pelos editores dos documentos.

Os avanços da tecnologia da informação e comunicação, a partir de 1960, proporcionaram novos meios para o suporte à informação, favorecendo o armazenamento em meio eletrônico e o acesso rápido proporcionado por ferramentas de busca. Neste contexto, a aplicação de tecnologia para o avanço da gestão de documentos deve ser considerada e atualmente, 
[...] os desafios que se colocam para os documentos eletrônicos conduzem a arquivística a uma aproximação cada vez mais intensa e profícua com as áreas da Administração, do Direito e da Informática, na busca de parâmetros científicos para o planejamento adequado da produção e para o tratamento dos documentos armazenados neste ambiente. (RODRIGUES, [2007?], p.1)

É importante lembrar que a aquisição de um software não se constitui numa condição "sine qua non" para o desenvolvimento da gestão de documentos, mas é, sem dúvida, um elemento facilitador, uma vez que permite ampliar significativamente as categorias de documentos gerenciadas (cada categoria poderá apresentar prazos de revisão e modelos específicos). Recursos de parametrização do sistema permitem a sinalização aos usuários da proximidade de revisão dos documentos, apresenta comandos para ações "em lote", como, por exemplo, a substituições de usuários em caso de desligamentos ou remanejamento entre setores, mas o grande avanço está na possibilidade de pesquisa por palavra-chave e a possibilidade de instituir usuários "genéricos" para o acesso de leitura. Ambas as opções, acesso e leitura, poderão ser realizadas de qualquer terminal disponível na instituição ou mesmo via web.

Mas se a aquisição do sistema for posterior ao desenvolvimento e à vigência dos documentos, é recomendada que, antes da aquisição, seja realizada uma análise técnica de aderência à estrutura já implementada. Um planejamento cuidadoso para a transição dos documentos do meio convencional para o eletrônico será requerido. É indicado que este planejamento contemple um cronograma com critérios para priorização as ações. Os critérios de priorização podem ser: categoria de documento; frequência de utilização dos documentos ou criticidade de ações contempladas pelos documentos. Há de se considerar os envolvidos no processo de edição, revisão e aprovação dos documentos, principalmente se houver diversas categorias de documentos, cada qual com seus requisitos e os editores selecionados forem os mesmos.

\section{Resultados}

A análise documental proposta deixou clara a contribuição dos modelos de avaliação externa para o estabelecimento de uma estrutura documental. Destaca-se como ponto forte um conjunto de documentos comum aos três modelos de avaliação estudados. Embora haja muitas diferenças entre os modelos e na dinâmica da avaliação entre as instituições citadas, observase uma homogeneidade de requisitos para uma estrutura documental mínima, conforme a Tabela 1.

Tabela 1 - Quadros 2, 3 e 4

\begin{tabular}{lcccc}
\hline & CQH & ONA & JCI & Total \\
\hline Processos & $\mathbf{8}$ & $\mathbf{5}$ & $\mathbf{1 3}$ & $\mathbf{2 6}$ \\
Procedimentos e/ou Manuais, normas e & $\mathbf{5}$ & $\mathbf{5}$ & $\mathbf{1 2}$ & $\mathbf{2 2}$ \\
rotinas/procedimentos & $\mathbf{2}$ & $\mathbf{5}$ & $\mathbf{1 3}$ & $\mathbf{2 0}$ \\
Diretrizes e/ou Políticas & $\mathbf{6}$ & $\mathbf{3}$ & $\mathbf{8}$ & $\mathbf{1 7}$ \\
Programas & $\mathbf{4}$ & $\mathbf{3}$ & $\mathbf{5}$ & $\mathbf{1 2}$ \\
Planos & $\mathbf{3}$ & $\mathbf{3}$ & $\mathbf{4}$ & $\mathbf{1 0}$ \\
Protocolos & $\mathbf{-}$ & $\mathbf{5}$ & $\mathbf{2}$ & $\mathbf{7}$ \\
Critérios técnicos e fluxos e/ou & & & & \\
Fluxogramas & & &
\end{tabular}




\begin{tabular}{lcccc}
\hline & CQH & ONA & JCI & Total \\
\hline Planos de Contingência & $\mathbf{1}$ & $\mathbf{5}$ & - & $\mathbf{6}$ \\
Normas e/ou Normas Éticas & $\mathbf{3}$ & - & $\mathbf{1}$ & $\mathbf{4}$ \\
Regimentos e/ou Estatutos/Regimentos & - & $\mathbf{1}$ & $\mathbf{1}$ & $\mathbf{2}$ \\
Listas / Critérios / Categorias & - & - & $\mathbf{1 0}$ & $\mathbf{1 0}$ \\
Interação formal & - & $\mathbf{6}$ & - & $\mathbf{6}$ \\
Planos de ação & $\mathbf{4}$ & - & - & $\mathbf{4}$ \\
Declaração de Direitos & - & - & $\mathbf{1}$ & $\mathbf{1}$ \\
\hline Total & $\mathbf{3 9}$ & $\mathbf{4 1}$ & $\mathbf{7 0}$ & $\mathbf{1 4 7}$ \\
\hline
\end{tabular}

Fonte: dados da pesquisa

As três metodologias consideram a documentação como um dos elementos necessários para se alcançar um padrão de qualidade nos serviços prestados pelas organizações de saúde, reconhecendo sua importância na consolidação da transparência da informação e como elemento indispensável para a melhoria dos processos assistenciais, apoio organizacional, e suporte ao diagnóstico e terapêutica.

Podemos deduzir que as organizações prestadoras de serviços hospitalares, ao desenvolverem uma estrutura documental com políticas, protocolos, normas, rotinas e procedimentos, estabelecem diretrizes, aperfeiçoam seus processos operacionais e de gestão, e desenvolvem melhor seus serviços, almejando evitar falhas e retrabalho, e, sensibilizando suas equipes para uma cultura de qualidade e segurança.

\section{Considerações Finais}

Objetivo deste artigo foi sistematizar a contribuição dos padrões, estabelecidos pelos modelos de avaliação externa de serviço, para a gestão de documentos em instituições prestadoras de serviços hospitalares, bem como ressaltar a opinião de especialistas para a importância deste tema na eficiência operacional.

Identificamos forte atuação das instituições acreditadoras no estabelecimento de requisitos de documentação, contribuindo, assim, para o avanço na gestão das organizações prestadoras de serviços hospitalares.

Para estabelecer a estrutura documental mínima, bastaria identificar as categorias de documentos mais requeridas nos modelos citados e envidar esforços para a concepção de um modelo de documento que atenda suas necessidades, e estágio de desenvolvimento do sistema da qualidade na instituição. Um instrumento de gestão que pode produzir resultados positivos nesta etapa é o benchmarking. Visitas com foco em gestão de documentos a instituições já acreditadas podem iluminar as ideias dos responsáveis pela definição da estrutura documental, bem como para a definição de modelos de documentos. Alguns serviços, pelo seu histórico de desenvolvimento, devem apresentar procedimentos documentados por força de legislação. São eles: Laboratório de Análises Clínicas, Serviço de Nutrição e Agência Transfusional. Os documentos elaborados por essas áreas podem ser referências para um padrão institucional. Cursos de atualização profissional para esta temática começam a surgir no horizonte das práticas de gestão e qualidade. 

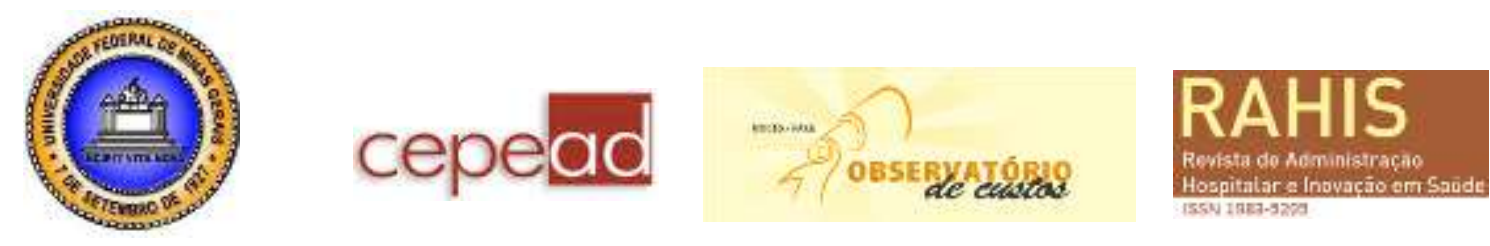

A aplicação de tecnologia (software) amplia significativamente o escopo da gestão de documentos, mas o desenvolvimento de um sistema de gestão da qualidade, e do estabelecimento de uma política de padronização e gerenciamento de documentos é um prérequisito para a utilização da tecnologia.

Todas as iniciativas reveladas neste estudo convergem, embora não sejam suficientes, para o estabelecimento de um programa de gestão de documentos, que deveria abranger, inclusive, a gestão dos impressos, pois, com as facilidades de criação e geração de cópias sem controle de versão, proporcionadas pelos programas de editoração de textos e planilhas eletrônicas disponíveis na maioria das instituições, estão muito aquém do conceito de eficiência necessário a toda e a qualquer instituição.

\section{Referências Bibliográficas}

BRASIL. Lei No 8.159, de 08 de janeiro de 1991. Dispõe sobre a política nacional de arquivos públicos e privados e dá outras providências. Diário Oficial da União República Federativa do Brasil, Imprensa Nacional, Brasília, DF, 9 jan. 1991. Seção 1, p. 455.

CAMARA, P.; TEIXEIRA, J. 40 Anos da FBAH: A História. - São Paulo: Apoena Produção Gráfica, 2011.

CASTILHO, N.T.; SILVA, C.E.S.; TURRIONI, J.B. Aprendizagem organizacional e gestão do conhecimento. In: XI SIMPEP, 2004, São Paulo, Disponível em: http://www.iem.unifei.edu.br/turrioni/congressos/simpep/2004/235-

Castilho_N_T_Aprendizagem_organizacional.pdf > Acesso em: 08 jul.2012.

COMPROMISSO COM A QUALIDADE HOSPITALAR - CQH. Roteiro de Visitas. São Paulo, versão 10-01. Disponível em:

<http://www.cqh.org.br/portal/pag/doc.php?p_ndoc=146> Acesso em: 10 dez. 2012.

COUTO R. C.; PEDROSA T.M.G. Hospital: Acreditação e Gestão em Saúde. 2. ed. - Rio de Janeiro: Guanabara Koogan, 2007.

Dicionário Brasileiro de Terminologia Arquivistica (2005), Disponível em:

<http://www.arquivonacional.gov.br/Media/Dicion\%20Term\%20Arquiv.pdf > Acesso em: 09 out. 2011.

JARDIM, J. M. O Conceito e a prática da gestão de documentos. ACERVO Revista do Arquivo Nacional, Rio de Janeiro, v.2, n.2, p.35-42, jul./dez. 1987.

ORGANIZAÇÃO NACIONAL DE ACREDITAÇÃO. Histórico. Disponível em: <https://www.ona.org.br/Pagina/23/Historico> Acesso em: 15 out. 2011.

ORGANIZAÇÃO NACIONAL DE ACREDITAÇÃO. Manual Brasileiro de Acreditação: Organizações Prestadoras de Serviços de Saúde. Brasília, versões 2006 e 2010.

RICHE, G.A.; MONTE ALTO, R. As Organizações que aprendem, segundo Peter Senge: "A Quinta Disciplina". In: Cadernos Discentes COPPEAD da UFRJ. Rio de Janeiro, n. 9, p. 36-55, 2001. 


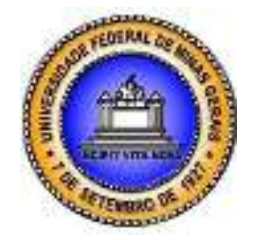

RODRIGUES, A.C. Gestão de documentos: uma abordagem conceitual. Disponível em: <http://www.ejef.tjmg.jus.br/home/files/publicacoes/gest_arqui/palestra_ana_celia_rodrigues. pdf > Acesso em: 9 out. 2011.

RONCAGLIO, C.; SZVARÇA, D. R.; BOJANOSKI, S. F. Arquivos, gestão de documentos e informação. Encontros Bibli: Revista Eletrônica de Biblioteconomia e Ciência da Informação, ano 9, n. esp, p. 1-13, 2004.

CONSÓRCIO BRASILEIRO DE ACREDITAÇÃO DE SISTEMAS E SERVIÇOS DE SAÚDE. Padrões de Acreditação da Joint Commission International para Hospitais. Rio de Janeiro, $4^{\mathrm{a}}$ ed. 2010.

SANTOS, A.P. Amnésia Organizacional: um Estudo de Caso Sobre a Memória na Administração Pública Federal. InCID: R. Ci. Inf. e Doc., Ribeirão Preto, v. 3, n.1, p. 3656, jan./jun. 2012.

SILVA, J. F.; ROCHA, A. Réplica 2 - A Estrutura Intelectual da Produção Científica de Alianças Estratégicas: Impressionismo ou Realismo? Revista de Administração Contemporânea, Curitiba, v. 14, n. 4, p. 744-751, jul./ago. 2010. 